\section{Replicating Surfaces}

Ron LHerault, Boston University lherault@bu.edu

Questions about how to replicate surfaces for microscopy keep arising on the microscopy listservers and elsewhere. There are commercial surface replication kits available, but for years I have used Caulk Reprosil, light body, a dental impression material to copy surface details such as on ceramics. The only time I had a problem was on a sample of my own, a sand dollar. It left an orange tint to the sand dollar because of the large, deep and plentiful pores.

After the Reprosil sets up, I box the edges with more Reprosil and then pour in Spurr's low viscosity embedding medium. When it sets, I have a positive to scan in an SEM that, up to around $1000 \mathrm{X}$ is almost impossible to distinguish from the original. I was taught that the best detail replication is achieved when one uses a gentle stream of dry air to push an initial thin coat of the Reprosil into the surface features.

This technique was originally published by $\mathrm{C}$. H. Pameijer and R. E. Stallard in J. Dent Res. Nov.-Dec., 1972, Vol 51(6), and has been more extensively discussed in other references such as Pameijer, C.H. 1979. Replication Techniques With New Dental Impression Materials in Combination With Different Negative Impression Materials. Scanning Electron Microscopy II. SEM Inc. pp $571-574$

\section{A Home Made Cryoadhesive for Mounting Specimens for CryoSEM}

Debby Sherman, Purdue University dsherman@purdue.edu

We have mixed carbon with Tissue Tec OCT cryoadhesive since the early 1980 s for mounting specimens on stubes for cryo-SEM. We have found it easier to make up the cryo-adhsive using weight rather than volume since the OCT is very viscous. The ethanol was added to help thin down the adhsive a bit for easier mixing and application. The formulation we use is:

- $0.4 \mathrm{~g}$ carbon powder (can be obtained from waste from sharpening carbon rods used for carbon coating)

- $2 \mathrm{~g} 95 \% \mathrm{ETOH}$

- $10 \mathrm{~g}$ OCT compound

Mix well and let sit for a few days prior to using.

\section{More Tips on Handling Specimens for CryoSEM}

\author{
Rosemary Walsh, Pennsylvania State University \\ rw9@psu.edu
}

I use an Oxford (now GATAN) C1500C system and have some experience imaging and analyzing cells grown in inserts, tissues, polymers, emulsions and foods. When possible I prefer unfixed tissue or fixed, cryoprotected cells.

In order to insure some success in removing surface ice, I blot excess fluid from a $2 \mathrm{~mm}$ sample, plunge into liquid nitrogen in a transport dewar located as close to the SEM sample preparation chamber as possible, cover and quickly press the nitrogen admit to open the chamber. After inserting the transfer holder onto the prep cryostage (which is at $-170^{\circ} \mathrm{C}$ ), I pull a vaccum, wait 2 minutes, fracture if needed, transfer onto the SEM coldstage, close the ball valve and immediately begin heating the stage. I prefer to do the transfer this way rather than into the liquid nitrogen slush where I run the risk of heavy ice contamination. Etching or sublimation can take 15 minutes for plant tissues and biopolymers and up to $\mathbf{4 5}$ minutes for emulsions and frozen foam foods (such as yogurt and ice cream).

If the sample size is large, e.g., 5 to $10 \mathrm{~mm}, 1$ attach it to the sample holder with a drop of graphite/OCT mix and immediately insert onto the sample prep stage (again, at $-170^{\circ} \mathrm{C}$ ) and proceed as outlined above. I've also modified this by progressively lowering the temperature using ice, then dry-ice, and finally the liquid nitrogen plunge.

One adaptation for dry-ice use is to cut out a hole in the side of a styrofoam container so that the transfer rod can be inserted into dry ice vapor. It is the only way to work with frozen samples.

It is important to minimize the sample size. I use gold planchets from an old Balzers freeze-fracture/freeze-etch system, as they have a tiny depression which holds $10 \mu \mathrm{L}$. This sits in a brass holder with an aluminum cap that holds the planchet in place. I invert a second planchet over the first with the sample, transfer both to the cryo-prep stage, wait two minutes, then fracture by touching a pick to the top planchet and proceed as above.

Another option is to use brass rivets. Our machine shop made 10 $\mathrm{mm}$ brass stubs with holes drilled to hold three rivets. I either drop liquid emulsion or scoop ice cream into them, cover with an inverted, cooled rivet, insert onto the cryo prep stage, pull a vacuum, tip the top rivet so that it fractures, then insert it into the SEM cryo-stage to begin sublimation and proceed as above.

It is imperative for us to schedule cryo-SEM work during lower humidity times. July and August are difficult. If I have to work then, I run a dehumidifier but it is still problematic.

I routinely mix a fresh batch of OCT cryo-mountant with a couple of drops of colloidal graphite, place a small drop onto the brass mount, and add a tiny wedge of filter paper to the drop along with the sample. I use the filter paper as a monitor for the sublimation/etch time. This has worked very well with pieces of tissue and polymers. It is important to make this fresh conductive adhesive, then keep it in a one $\mathrm{mL}$ microfuge tube and apply with a wooden pick or with a disposable 1 cc syringe.

Examples of the results we get using these protocols are shown in Figure 1. This specimen is the control, which is compared to similar tissue of mutant strains of Arabidopsis thaliana for plant genetics and plant developmental biology. Because cell walls in all $A$. thaliana tissues are thin, attempts to retain ultrastructure using conventional SEM preparation methods of fixation, dehydration, and critical point drying are problematical, so we prefer cryomethods.

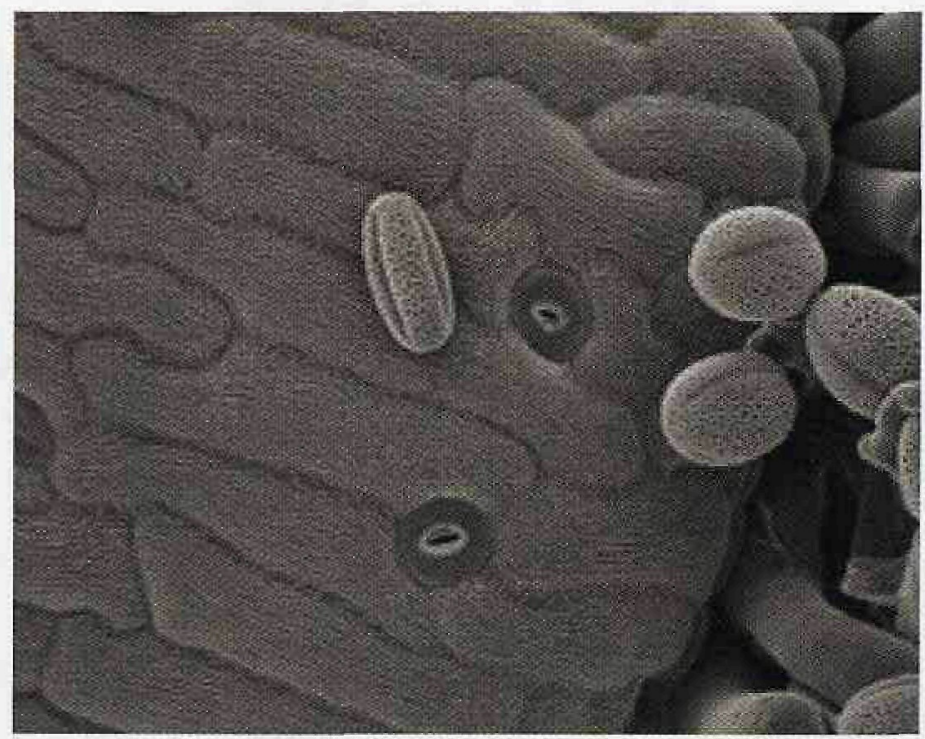

Arabidopsis thaliana; wild type. Gyneocium surface with papillae and pollen. $-196^{\circ} \mathrm{C}$, etched $15 \mathrm{~min}$. Au coated, $10 \mathrm{kV}, 750 \mathrm{X}$ 